\title{
Prolapso de Tuba Uterina após Histerectomia Vaginal: Relato de Caso
}

\author{
Fallopian Tube Prolapse after Vaginal Hysterectomy: a Case Report \\ Maurício B. Noviello, Agnaldo L. Silva-Filho, Admário S. Santos-Filho, \\ Eduardo B. Cândido, Sérgio A. Triginelli
}

\begin{abstract}
RESUM0
O prolapso de tuba uterina é complicação rara após histerectomia, com aproximadamente 80 casos descritos na literatura. A sintomatologia é inespecífica, podendo incluir sangramento genital, dispareunia e dor pélvica crônica. O diagnóstico diferencial deve ser feito com granuloma de cúpula vaginal e carcinoma de vagina. O tratamento deve ser individualizado, podendo ser realizado por via vaginal, abdominal ou laparoscópica. Relatamos o caso de uma paciente, 47 anos, com miomatose uterina, submetida a histerectomia vaginal, evoluindo com prolapso de tuba uterina após 11 meses de pós-operatório. O exame especular evidenciava lesão vegetante, friável e sangrante localizada na cúpula vaginal. Esses achados clínicos sugeriam o diagnóstico de prolapso de tuba uterina. A paciente foi submetida a nova intervenção cirúrgica, com ressecção da tuba uterina por via vaginal. O exame natomopatológico confirmou o diagnóstico e a paciente evoluiu com remissão completa da sintomatologia.
\end{abstract}

PALAVRAS-CHAVE: Tuba uterina. Histerectomia. Histerectomia vaginal. Complicações pósoperatórias.

\section{Introdução}

A técnica de histerectomia vaginal, descrita por Heaney 1934, apresenta algumas vantagens em relação à via abdominal. A via vaginal proporciona uma menor manipulação de alças intestinais, um pós-operatório menos doloroso, além de um menor tempo de internação e convalescência. Outra vantagem é a possibilidade de correção pela mesma via das distopias genitais associadas ${ }^{12}$.

O prolapso de tuba uterina é complicação rara, descrita desde 1902, com cerca de 80 casos relatados na literatura ${ }^{3}$. Essa complicação é mais freqüente quando a histerectomia é realizada por via vaginal $^{4,5}$. Os sintomas são inespecíficos, sendo o diagnóstico realizado pela visualização de um teci-

Departamento de Ginecologia e Obstetrícia da Faculdade de Medicina da Universidade Federal de Minas Gerais (UFMG) e CENAGIN (Centro Avançado de Cirurgia Ginecológica). Hospital das Clínicas da Universidade Federal de Minas Gerais.

Correspondência:

Agnaldo Lopes da Silva Filho

Avenida Pasteur, 89/1310 - Bairro Santa Efigênia

30150-290 - Belo Horizonte - MG

e-mail: agsilvaf@terra.com.br do hiperemiado, friável e sangrante ao exame ginecológico ${ }^{5}$. O tratamento deve ser individualizado, podendo ser realizado por via vaginal, abdominal ou laparoscópica.

Relatamos o caso de uma paciente que apresentou essa complicação rara após histerectomia vaginal, cujo diagnóstico e tratamento adequados possibilitaram a completa remissão dos sintomas.

\section{Relato do caso}

Paciente com 47 anos, G5P3A2, e antecedentes de três cesarianas prévias e sem doenças associadas. No primeiro atendimento apresentava queixa de sangramento uterino anormal e anemia sem melhora com tratamento hormonal e suplementação de ferro. O exame clínico evidenciava um útero não prolapsado e aumentado de volume, além de uma boa mobilidade uterina, amplitude vaginal e arco púbico adequados. A ultra-sonografia mostrava útero miomatoso com volume estimado de $220 \mathrm{~cm}^{3}$, sem alterações nos anexos ou endométrio. 
Foi realizada histerectomia vaginal sob raquianestesia no Hospital das Clínicas da UFMG em dezembro de 2001. Foi empregada a técnica de Heaney de histerectomia vaginal, não sendo realizada anexectomia. Não foi realizada peritonização e a síntese da cúpula vaginal foi feita com sutura contínua com Vicryl $1{ }^{\circledR}$. A cirurgia transcorreu sem intercorrências, com duração de 60 minutos. A paciente não apresentou complicações no pós-operatório imediato e recebeu alta hospitalar após 24 horas de internação. O seguimento da paciente foi realizado no $15^{\circ}$ e $30^{\circ}$ dia de pós-operatório e, a partir de então, semestralmente.

Após 11 meses da cirurgia a paciente iniciou quadro de secreção vaginal amarelada e sangramento após as relações sexuais. O exame especular evidenciou a presença de uma lesão vegetante com dois centímetros de diâmetro, friável e sangrante na cúpula vaginal. Foi também identificado um defeito com cerca de um centímetro de diâmetro no local da rafia na cúpula vaginal. A tração da estrutura herniada com pinça era dolorosa e mostrava sua continuidade, que se tornava tubular, para a cavidade peritoneal (Figura 1). Esses achados clínicos sugeriam fortemente o diagnóstico de prolapso de tuba uterina, não sendo realizada biópsia, propondo-se tratamento cirúrgico.

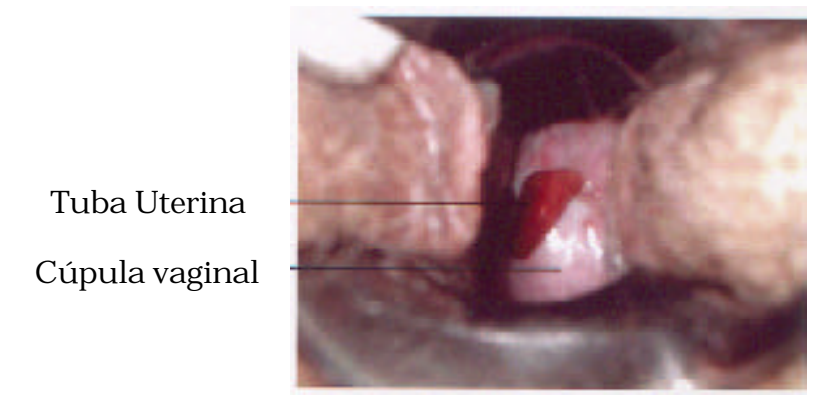

Figura 1 - Exame especular evidenciando o prolapso da tuba uterina pela cúpula vaginal.

Em fevereiro de 2003 a paciente foi submetida a nova intervenção cirúrgica, sob anestesia geral venosa. A paciente foi colocada em posição de litotomia, sendo realizada anti-sepsia e colocação dos campos cirúrgicos. Após colocação de espéculo vaginal foi realizada uma ampliação do defeito da cúpula vaginal, tração da tuba uterina com pinça de Allis e feita dissecção com tesoura até a sua porção ístmica. Realizou-se clampeamento e ressecção da tuba uterina esquerda. Após revisão da hemostasia foi realizada sutura da mucosa vaginal com Vicryl $1{ }^{\circledast}$ em pontos separados. O pós-operatório evoluiu sem intercorrências e a paciente recebeu alta hospitalar 12 horas após a intervenção.

O exame natomopatológico da peça cirúrgica evidenciou uma estrutura tubular, macia e friável medindo cinco centímetros. A microscopia identificou áreas de inflamação crônica sem atipias nucleares e confirmou o diagnóstico de tuba uterina. A paciente evoluiu com melhora da sintomatologia e exame clínico mostrando cúpula vaginal íntegra e bem cicatrizada após seis meses de seguimento.

\section{Discussão}

A histerectomia vaginal sem prolapso está associada a uma menor incidência de complicações que a histerectomia abdominal ${ }^{6}$. Um estudo multicêntrico, avaliando 1851 pacientes submetidas a histerectomia via vaginal e abdominal, mostrou taxas de complicações de 24 e 42,8\%, respectivamente $^{6}$. O prolapso de tuba uterina foi evidenciado em 17 casos em estudo no qual foram avaliadas 8444 pacientes submetidas a histerectomia, não havendo diferenças em relação à histerectomia abdominal e à vaginal ${ }^{4,7}$. No entanto, o risco de prolapso de tuba uterina é três vezes superior quando a histerectomia é realizada pela via vaginal $^{5}$. Dos 80 casos de prolapso de tuba uterina relatados na literatura, a via vaginal era a cirurgia antecedente em $66,5 \%$ dos casos e a via abdominal em 32\%, sendo a histerectomia vaginal assistida por laparoscopia em 15\% dos casos ${ }^{5}$. Foi descrito o caso de uma paciente que evoluiu com essa complicação após ser submetida a uma histerectomia subtotal por via abdominal ${ }^{7}$.

O prolapso de tuba uterina é decorrente da existência de um defeito na cúpula vaginal e conseqüente comunicação entre a cavidade peritoneal e a vagina. O período de ocorrência dessa complicação pode variar de 15 dias a 28 anos após a cirurgia primária ${ }^{7,8}$. Constituem fatores de risco para o prolapso de tuba uterina a utilização de drenos vaginais, deiscência da sutura vaginal, desnutrição e baixo nível socioeconômico ${ }^{4,7,9}$. Os hematomas e abscessos pélvicos podem prejudicar a cicatrização da cúpula vaginal ou drenarem espontaneamente para a vagina, criando um pertuito para herniação tubária ${ }^{5,7,8}$.

A sintomatologia do prolapso de tuba uterina quando presente é inespecífica, podendo incluir sangramento vaginal, leucorréia, dispareunia e dor pélvica crônica ${ }^{4,9,10}$. O diagnóstico é realizado por meio do exame especular e visualização de uma lesão polipóide, avermelhada e vegetante na cúpula vaginal. A manipulação da lesão é geralmente dolorosa e sangrante ${ }^{11}$. O diagnóstico diferencial deve ser feito com granuloma de cúpula vaginal, adenose vaginal, endometriose, cisto paravaginal e fístula vésico-vaginal, além do carcinoma de vagina $^{3,4,12}$. O diagnóstico definitivo é realizado pelo 
exame histopatológico da lesão, que pode ser inconclusivo devido à inflamação aguda ou crônica alterando a histologia do tecido tubário. A presença de atipias celulares provenientes de uma reação inflamatória associada pode sugerir o diagnóstico de carcinoma vaginal primário9.

O tratamento cirúrgico constitui a abordagem de escolha no prolapso de tuba uterina ${ }^{3}$. A cauterização, elétrica ou química, foi utilizada em pacientes com diagnóstico errôneo de tecido de granulação, apresentando resultados insatisfatórios. A abordagem cirúrgica pode ser realizada por via vaginal, abdominal ou laparoscópica. Em 1986 foi descrita uma técnica de salpingectomia total por via vaginal para tratamento do prolapso de tuba uterina. O procedimento consiste em incisão elíptica ao redor da tuba prolapsada, dissecação até a porção ístmica, secção e ligadura, seguido de rafia da mucosa vaginal ${ }^{13}$. Algumas pacientes com dor pélvica e dispareunia podem se beneficiar da avaliação vídeo-laparoscópica associada à via vaginal. Essa abordagem proporciona maior segurança na visualização direta da lesão e lise de aderências ${ }^{4,14}$. O tratamento ambulatorial pode ser realizado por meio da utilização de um endoloop na base da tuba prolapsada ${ }^{15}$.

A salpingectomia bilateral, realizada no momento da histerectomia, a peritonização pélvica e a não utilização de drenos vaginais constituem medidas importantes na prevenção dessa complicação ${ }^{3}$. Os resultados do tratamento cirúrgico do prolapso de tuba uterina, independente da via cirúrgica adotada, são satisfatórios, com melhora da sintomatologia, sendo a recidiva rara $^{5}$.

\section{ABSTRACT}

Fallopian tube prolapse is a rare complication after hysterectomy, with approximately 80 cases described in the literature. The symptoms are nonspecific including vaginal bleeding, dyspareunia and chronic pelvic pain. The differential diagnosis must be done with granulation tissue of the vaginal cuff and vaginal carcinoma. The treatment should be individualized, and is possible to be done by vaginal, abdominal or laparoscopic approach. We report a case of a 47-year-old woman with myoma who was submitted to a vaginal hysterectomy and evolved with fallopian tube prolapse 11 months after surgery. Specular examination showed a fungating, friable and bleeding lesion at the vaginal cuff. The clinical findings suggested the diagnosis offallopian tube prolapse. The patient was submitted to a new surgical intervention with resection of the left fallopian tube. The histologic examination confirmed the diagnosis and the patient evolved with complete remission of the symptoms.

KEYWORD: Fallopian tube. Hysterectomy. Vaginal hysterectomy. Postoperative complications.

\section{Referências}

1. Figuerêdo Netto $\mathrm{O}$, Figuerêdo $\mathrm{O}$, Pinotti J. Histerectomia vaginal em pacientes sem prolapso uterino: análise de 150 casos. Rev Bras Ginecol Obstet 1997; 19:267-74.

2. Heaney NS. Vaginal hysterectomy: its indications and technique. Am J Surg 1940; 48:284-8.

3. Piacenza JM, Salsano F. Post-hysterectomy fallopian tube prolapse. Eur J Obstet Gynecol Reprod Biol 2001; 98:253-5.

4. Ramin SM, Ramin KD, Hemsell DL. Fallopian tube prolapse after hysterectomy. South Med J 1999; 92:963-6.

5. Candiani GB, Candiani M. Post-hysterectomy fallopian tube herniation. A report of two cases. J Reprod Med 1996; 41:915-20.

6. Dicker RC, Greenspan JR, Strauss LT, et al. Complications of abdominal and vaginal hysterectomy among women of reproductive age in the United States. The Collaborative Review of Sterilization. Am J Obstet Gynecol 1982; 144:841-8.

7. de Kroon CD, Bergman I, Westenberg S, van Eyk H, Thurkow AL. Prolapse of the uterine tube after subtotal hysterectomy. BJOG 2003; 110:333-4.

8. Wheelock JB, Schneider V, Goplerud DR. Prolapsed fallopian tube masquerading as adenocarcinoma of the vagina in a postmenopausal woman. Gynecol Oncol 1985; 21:369-75.

9. Muntz HG, Falkenberry S, Fuller AF Jr. Fallopian tube prolapse after hysterectomy. A report of two cases. J Reprod Med 1988; 33:467-9.

10.Lavy Y, Hamani Y, Lev-Sagie A. Fallopian tube prolapse after hysterectomy. Harefuah 2002; 141:530-1, 578.

11.Bilodeau B. Intravaginal prolapse of the fallopian tube following vaginal hysterectomy. Am J Obstet Gynecol 1982; 143:970-1.

12.Jensen H, Rasmussen KL. Prolapse of the salpinx after hysterectomy. Ugeskr Laeger 1996; 158:5040-1.

13.Wetchler SJ, Hurt WG. A technique for surgical correction of fallopian tube prolapse. Obstet Gynecol 1986; 67:747-9.

14.Hernandez CR, Howard FM. Management of tubal prolapse after hysterectomy. J Am Assoc Gynecol Laparosc 1998; 5:59-62.

15.Harris RL, Speights SE, Moore JR Jr, Hampton HL. Fallopian tube prolapse: a noninvasive technique for correction. J Pelvic Surg 1995; 1:47-49.

Recebido em: 29/8/2003 Aceito com modificações em: 3/11/2003 